\title{
BLOCKCHAIN IN IoT AND PROJECT MANAGEMENT
}

\author{
Eugene Amoah, Texas A\&M University-Kingsville, eugene.amoah@students.tamuk.edu \\ Joon-Yeoul Oh, Texas A\&M University-Kingsville, joon-yeoul.oh@ tamuk.edu
}

\begin{abstract}
Blockchain technology establishes an ecosystem in IoT that is creditworthy among the participants for high-security requirements. However, there has not been comprehensive researches done on the blockchain in IoT nor its application potential in Project management. The objectives of this research are to investigate the role of blockchain in IoT and to identify prospective applications of blockchain technology in project management. Both IoT and project management require the secured data transmission and effective yet efficient data management. The blockchain-based technology can be applied in the areas of IoT and project management because it can provide immutability, transparency between users in a complex system. This research needs to be extended to build a prototype open-source blockchain-based project management application.
\end{abstract}

Keywords: Blockchain Technology, IoT, Project Management

\section{INTRODUCTION}

The emergence of blockchain work was first published in 1991 by Stuart Haber and W. Scott Stornetta and later applied as a Hash-Cash-like method to timestamp blocks (Narayanan et al., 2016). This method of application, which is commonly known as Bitcoin, was first published by Nakamoto (2018), and it was implemented as opensource software for some transactions. Over a decade, the blockchain-based technologies and their applications became a driver of the next industrial revolution (Pournader et al., 2020).

A blockchain is a distributed ledger technology (DLT) that provides the framework for developing a decentralized, secure, and reliable operation (Pop et al., 2020). This technology provides several advantages, such as transparency, openness, traceability by joining several computer science disciplines such as cryptography, data structures, and consensus algorithms (Zhao at al., 2017). The basic infrastructure of the blockchain is to provide enough creditworthy data-level storage and operations (Li, 2019). After smart contracts were introduced by Szabo (1997), blockchain gained its significant benefit. The data transactions were automatically executed using script according to predefined rules (Christidis \& Devetsikiotis, 2016; Cong \& He, 2019). Thus, a blockchain can be summarized as smart contracts in the form of decentralized, distributed, and a public ledger whose framework allows the recording of transactions across many peered nodes so that these records cannot be retroactively altered without altering subsequent blocks and its entire network (Li, 2019).

IoT has seen an exponential increase in its services connecting about 30 billion devices (Lund et al., 2014). It allows the interconnection of multiple smart electronic devices in an environment (Wang et al., 2019). However, IoT devices are susceptible to multiple security and privacy issues, which have either been long overlooked or ignored (Wurm et al., 2016). It is critical to address vulnerabilities such as high implementation cost and current incompatible security methods with its decentralized topology (Brody \& Pureswaran, 2014). IoT devices continuously retrieve information about their surroundings and exchange the information with one another and their cloud servers through data aggregation systems (Mazzei et al., 2020). The visibility of such information, sometimes sensitive, gathered by these IoT devices poses concerns of potential privacy breaches (Roman et al., 2013). Microsoft (2009) reported in a survey that $75 \%$ of its respondents who were classified as IoT adopters expressed concerns about the technology's security issues. The information obtained by IoT devices is digitized and transmitted but its reliability is a big challenge (Reyna et al., 2018). Current security solutions for information systems are mostly applicable to centralized systems, unlike IoT platforms. These security concerns raised about IoT call for a secured Distributed Ledger system, such as blockchain, that ensures data obtained from IoT platforms are secured and immutable (Mazzei et al., 2020). However, there has been limited research on blockchain and its applications after blockchain’s explosive awareness was created through cryptocurrencies in 2008. 
Table 1 above shows the evolution of cryptocurrency over time. The early blockchain years from 1991 -2008 saw the birth of the blockchain proof of concept by Stuart Haber \& W. Scott Stornetta (1991). In 1991, the authors envisioned the development of documents which were consisted of a cryptographically secured chain of blocks that were tamper-free. Bayer et al. (1993) improved the efficiency of the earlier work of Stuart Haber \& W. Scott Stornetta (1991) by incorporating Merkle trees enabling the collection of multiple documents in a single block.

Table 1. Cryptocurrency Evolution Timeline

\begin{tabular}{|c|c|c|c|c|}
\hline Timeline & Blockchain & Bitcoin & Ethereum & NEO \\
\hline 1991-2008 & $\begin{array}{l}\text { Stuart Haber and } \\
\text { Scott Stornetta work } \\
\text { on the first } \\
\text { Blockchain }\end{array}$ & & & \\
\hline 2009 & & $\begin{array}{l}\text { Satoshi Nakamoto releases } \\
\text { Bitcoin white paper }\end{array}$ & & \\
\hline 2010 & & $\begin{array}{l}\text { The first Bitcoin purchase } \\
\text { 10,000BTC }\end{array}$ & & \\
\hline 2013 & & $\begin{array}{l}\text { Bitcoin marketplace } \\
\text { surpasses \$1 Billion }\end{array}$ & $\begin{array}{l}\text { Vitalik Buterin releases } \\
\text { Ethereum white paper }\end{array}$ & \\
\hline \multicolumn{5}{|c|}{$\begin{array}{l}\text { Ethereum Blockchain } \\
\text { funded by Crowdsale }\end{array}$} \\
\hline 2014 & $\begin{array}{l}\text { Blockchain } \\
\text { Technology R3 is } \\
\text { formed }\end{array}$ & & & \\
\hline 2014 & & & & $\begin{array}{l}\text { NEO project } \\
\text { launched as } \\
\text { Antshares by Da } \\
\text { Hongfei and Erik } \\
\text { Zhang }\end{array}$ \\
\hline 2015 & & & $\begin{array}{l}\text { Ethereum Second } \\
\text { Blockchain unveiled }\end{array}$ & \\
\hline 2015 & $\begin{array}{l}\text { Linux Foundation } \\
\text { unveils Hyperledger }\end{array}$ & & & \\
\hline 2017 & $\begin{array}{l}\text { EOS.IO is unveiled by } \\
\text { block one as a new } \\
\text { blockchain protocol }\end{array}$ & & & \\
\hline 2015-presen & \multicolumn{4}{|c|}{ Blockchain Technology evolving continuously } \\
\hline
\end{tabular}

In the period from 2008 to 2013, Nakamoto (2008) released the first white paper about the first phase of a working blockchain in the form of Bitcoin. He implemented it as a peer-to-peer system where the genesis (first) block of chains was formed. In the period from 2013 to 2015, the next evolution of blockchain was spearheaded by Buterin (2014), who developed Ethereum. The Ethereum differentiated itself from blockchain by adding an extra feature that allows users to record other assets such as contracts and slogans. This ensured the development of decentralized applications and eventually smart contracts. From 2015 to 2018, the third evolution of the blockchain period, the blockchain-based applications, such as Hyperledger by Linux, EOS, NEO launched in china, and IOTA were developed (Goyal, 2018).

Blockchain technology can be applied not only in the field of financial transactions but also in other applications, such as smart energy and grids, digital identity schemes, biometrics, healthcare, supply chain (Pan et al., 2019). 


\section{Issues in Information Systems \\ Volume $X$, Issue $X$, pp. $x$ - $x$, Year}

Many industries use IoT enabled devices in which blockchain can be exploited to improve their supply chain function (Pournader et al., 2020). In biometrics, advanced encryption schemes including homophonic encryption and multi-party computation (MPC) are being employed as a non-standard protection technique to manage biometric data in a public blockchain framework (Delgado-Mohatar et al., 2020).

Figure 1 describes a simplified illustration of how a blockchain works. The key properties that make a blockchain superior are peer-to-peer transactions, distributed ledger, cryptographically secured, "add-only" and decentralized data (IP Specialist, 2019).

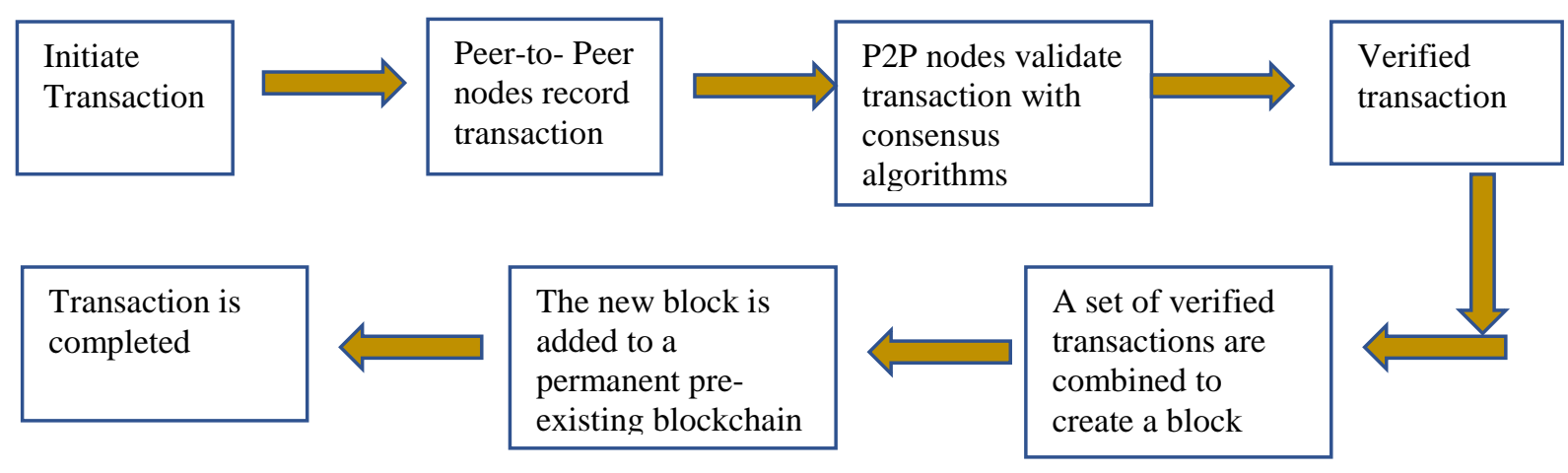

Figure 1. Process of Blockchain Technology

A transaction is initiated by a node and digitally signed with a private key. The transaction is propagated using a gossip protocol to peers for validation based on preset criteria. After validation, the transaction is included in a block, which is a set of data, and propagated onto the network. The newly created block is now part of the ledger and subsequent block links itself cryptographically back into this block. Transactions are reconfirmed with every addition of a new block, and a chain of blocks is formed.

The data integrity and trust are the long standing challenges in the business and financial sectors. Some of the challenges were settled by applying the blockchain's disruptive technology. This paper, first of all, investigates the blockchain-based applications and blockchain-based IoT applications. This paper also discusses about the potential adoption of the blockchain technology in solving similar issues in project management. Finally, the feasibility and challenges of the blockchain application as applying in the field of the project management are discussed.

\section{BLOCKCHAIN APPLICATIONS}

\section{Power Systems}

Since its introduction to the world, the application of blockchains has gone beyond the financial sector and into other areas such as power systems. Three main electrical power applications are electrical energy trading, renewable energy certification, and demand response tracing (Di Silvestre et al., 2020). Most of its application in the energy field are aimed at Peer-to-Peer energy trading (Jogunola et al., 2017). However, depending on the parties involved, the blockchain application may change in the other power system fields, such as electric vehicles (Huang et al., 2018), producers or prosumers, and energy storage system (Pop et al., 2018). In electrical power trading, it is used to facilitate machine-to-machine interactions so that the power producers and prosumers can create a small-scale market for exchange (Perrons \& Cosby, 2020). The main property that all current blockchain applications and future models on blockchain seek to leverage the property of transparency (Maesa \& Mori, 2020). Maesa and Mori (2020) also pointed out that this key property, transparency, is based on immutability, tamper resistance, and persistence of blockchain's data storage ability. The key property ensures the suitability of all applications built on blockchains. 


\section{Geo-energy}

Feng et al. (2020) identified the potential use of blockchain to solve problems related to physical assets, even though blockchain has been mainly used to solve the problems related to digital assets. The authors hypothesized how blockchain could be used to eventually deliver solutions in the geo-energy domain, in which the realistic short-term expectations in this field would be to tackle key areas of the sector. Blockchains are currently being used to create shared consensus about the progress of drilling campaigns (Rassenfoss, 2018). Lacity (2018) proposed that geoenergy market transactions can be managed using the blockchain technology.

\section{Maritime Shipping Digitization}

Global logistics has become an increasing necessity in today’s turbulent environment of international trade and global partnerships (Chang and Lai, 2017). Due to the complication of global trading, the maritime industry needs to deal with high volumes of paperwork resulting in slow processing of documentations (Lieber, 2017). Slowing processing time due to many documentation causes the loss of goods, misplacements as well as poor services (Yang, 2019). It is estimated that about $15 \%$ to $50 \%$ of transportation costs in the global container shipping industry is due to the paperwork processing time (Groenfeldt, 2017). Maersk and other major transshipment companies have worked hard to digitize records and transactions through the blockchain (Nguyen, 2019). Blockchain technology allows not only tracking of shipment and delivery time but a wider range of global logistics partners such as shippers, ocean freight forwarders, shipping carriers, terminal operators, and customs offices. This does not only enhance transparency between all parties involved but improves scheduling activities carried out by the various partners within a supply chain (Yang, 2019).

\section{Agri-Food Traceability}

To sustain agri-food chains, it is critical to track and authenticate the information throughout the whole food supply chain (Galvez et al., 2018; Olsen and Borit, 2018, Zhao et al., 2017). Feng et al. (2020) identified the potential of using blockchain technology-based applications to solve such issues currently faced in food traceability. The authors developed a framework with specific characteristics and functionalities for food traceability management and explored development and implementation strategies needed to tackle challenges faced by researchers and practitioners of food traceability. Agri-transactions, first of all, will be coordinated using blockchain and the data will be propagated between stakeholders (Galvez et al., 2018). The initiated agri-transactions would then be validated using the Merkle tree (Kshetri, 2018). The agriculture goods flow will be recorded with IoT platforms and sensors such as RFID or barcodes (Fernández-Caramés et al., 2018). The transaction events are recorded and included in the blockchain on the product supply chain (Khan et al., 2018). Stakeholders in the supply chain will add new information in the blockchain once a consensus is reached to ensure the information is reliable (Andoni et al., 2019). To improve the security of information, a block of historical data cannot be tampered after it is added to the chain (Thakur \& Donnelly, 2010).

\section{Electronic Voting}

Electronic voting refers to any type and any form of voting system where ballots are cast through an electronic system (Ali \& Murray, 2016). Proper implementation of e-voting can solve common fraud, provide a convenience for the voters through limited direct contact and accessibility, and possibly reduce the cost of the electoral system (International Institute of Democracy and Electoral Assistance, 2011). However, the use of e-voting can make some issues including the lack of transparency, risk of manipulation by insiders with privileged access, and reduced level of control by administrations of the election. Maesa and Mori (2020) outlined an e-voting system integrated with blockchain frameworks to solve some underlying concerns in the use of e-voting, where a bitcoin-style cryptocurrency oriented blockchain is adopted by first verifying voters. The voters cast their preferred vote using a voting token, then the total tally per candidate is counted and validated through blockchain's validation system. 


\section{Healthcare}

The healthcare industry is a classic case that deals with a lot of privacy requirements with the advent of technology and mobile devices (Karafiloski \& Mishev, 2017). Some key requirements for the adoption of technology in the healthcare sector are authentication, interoperability, data transfer, and data sharing (Karafiloski \& Mishev, 2017). Moreover, in today's digital age, most national health systems are trying to collect all personal health-related data in a unique electronic record, called Electronic Health Record (EHR) (Maesa \& Mori, 2020). The records require a high level of protection to prevent breaches from unauthorized parties (Boban, 2016). Blockchain technology offers strong potential applications in the healthcare system by providing security, integrity, availability, and authentication principle due to its ledger and block-based infrastructure to help meet the requirements as well as solve issues of interoperability, patient empowerment, and secured record sharing (Karafiloski \& Mishev, 2017).

\section{IoT AND BLOCKCHAIN}

\section{IoT and IIoT}

Despite the increase in proof of concepts and demos with blockchain applications (Pawczuk et al., 2018), "the real industrial use of the Blockchain technology is still a chimera" as quoted by Mazzei et al. (2020). The introduction of the Internet of Things (IoT) and its application to industries (IIoT) reshape traditional thinking and allow the interconnection of objects in a network environment (Sengupta et al., 2020). However, the risks of privacy and security vulnerabilities have been of rising concern (Wang et al., 2019). The Internet of Things refers to any number of physical objects which are interconnected to one another through the internet and they continuously exchange data obtained from their surroundings with one another (Atzori et al., 2010). Fundamental characteristics of blockchain technology, such as decentralization and information disclosure, have been proposed a solution to the growing concern of risk in IoT and IIoT, and it has been shown that the combined technologies can pave the way for novel models and IoT applications (Wang et. al., 2019). Wang et al. (2019) also insist that IoT, IIoT, and blockchain can be combined to promote platform functions in food industry logistics management, even though some limitations are present with the combination. The growing number of transactions due to the combination increases complexity in the mining algorithm, so it requires a higher energy consumption to perform the transactions and the blockchain designs become less energy efficient (Wang et al, 2019). Another limitation hampering the use of the blockchain applications in IoT is to identify the participating devices in the blockchain using its public key, which presents a complicated privacy issue (Meiklejohn, 2013).

\section{Security/Privacy Challenges in IoT}

IoT infrastructure consists of three physical layers network and application and therefore implies an interconnection of multiple smart devices that interact with one another to perform a function through sensors and integration of technologies, such as IoT-Cloud or IoT-fog-Cloud (Mohanta et al., 2020). For the reliable IoT applications, some security issues, such as machine-to-machine communication, real-time monitoring, and data privacy, need to be addressed (Al-Hasnawi et al., 2019; Casola et al., 2019; Chen \& Lien, 2014). Common attacks on three layers of IoT include the intrusion detection system (IDS), power analysis attack, wormhole attack, and man-in-the-middle attack (Almiani et al., 2019; Deshmukh-Bhosale \& Sonavane, 2019; Li et al., 2017; Moon et al., 2018).

\section{Blockchain Solution to Challenges in IoT}

Several solutions to IoT applications were addressed by researchers from 2018 through 2020. One of the solutions is to use a distributed ledger-based blockchain, which can be used to solve IoT privacy issues, such as spoofing and false authentication (Sadique et al., 2018). Also, to secure datasets sharing in an IoT environment, blockchain-based compromised firmware detection was explored by Banerjee, Lee \& Choo (2018). To enhance the security of IoT systems, a device classification can be explored by applying machine learning algorithms on the blockchain data, which is secured in its network (Dorri et al., 2019). Prada-Delgado et al. (2020) proposed to combine the blockchain with IoT devices that exploit the characteristics of memory chips, which use cryptographic secret as an anticounterfeiting approach to verify the devices' identities reliably. Since the blockchains are essentially distributed 
ledgers to validate nodes for its transactions, the trust management of multiple nodes for IoT applications are required to make more efficient to improve the decision making of the IoT (Casado-Vara et al., 2019).

\section{BLOCKCHAIN TECHNOLOGY IN PROJECT MANAGEMENT}

The blockchain technology possessed a unique set of features such as anonymity, security, accountability, robustness, and transparency that encouraged its application in the various sectors including smart contracts (Hewavitharana et al., 2019). Thus, blockchain technology can be applied in the area of project management, which requires tracking long-standing issues.

Norris (2019) proposed the management of scientific research projects, in which their solutions are easily applicable to other types of projects. The traditional scientific project management exists within independent departments (Bai et al., 2018), which their connections are surrounded in rigidity and necessity (Aiping \& Dongliang, 2016). Due to such inflexibility, there have been occurrences of academic misconduct such as forgery, misappropriation of funds, and repeated publications in the course of such projects (Zheng \& Bai, 2018). Bai et al. (2018) proposed to the building of an innovative union blockchain-based project management tool that incorporates in various layers, such as data layer, network layer, consensus layer, incentive layer, and contract layer, to tackle the problems. The blockchain framework can be applied to other areas of project management. In the projects of the construction industries, where use building, information, and modeling (BIM), a blockchain-based BIM pack can facilitate between stakeholders, such as the architectural firm, civil engineers, suppliers, and others, to provide an immutable record of the projects' data (Norris, 2019).

Smart contracts on a network are the compiled computer protocols that embed the terms and conditions (the source code) of a contract (Gronbaek, 2016). Smart contracts could potentially be used to manage independent tasks in project management workflows (Norris, 2019). The blockchain-based smart contracts, for example, can be used in case of project failure, which is associated with legal consequences (Turk \& Klinc, 2017). In construction projects, the blockchain-based smart contract provides a useful tool for managing and recording changes of the BIM model as negotiating editing privileges between stakeholders (Lohry \& Bodell, 2015). Ultimately, the status reports and automatic payments can be made for subcontractors through smart contracts (Lawrence, 2019).

The role of the Project Management Office (PMO) can be altered for growing in the usage of blockchain-based applications. According to the report released by the Blockchain Research Institute in collaboration with Brightline Initiative in 2019, the new roles of PMO include creating and managing digital records, exchanging of digital assets, and reinforcing acceptable performances (Giffen, 2019). The Project Management Offices can have benefited from the blockchain-based applications for automating record-keeping and coordinating the tasks between stakeholders. These benefits help PMO to manage project scope, costs, and schedules more efficiently yet effectively.

\section{CONCLUSION}

Blockchain has been a disruptive innovation and it has provided the industrial alternatives in many industries as a generic technology platform over the last decade. Despite the lack of researches and its practical adoptions in the project management field, this paper aimed to fill this gap by identifying and analyzing the current role of blockchain in IoT and the application of blockchain technology in the new industrial revolution wave. This research also examined the possibility of blockchain technology to solve long-standing challenges in the project management field. In conclusion, the blockchain technology provided prominent features such as persistence, immutability, and security, so that its technology can easily be adapted to IoT applications and the security challenges in IoT. Similarly, blockchain technology can be applied to project deliverable tracking, project transaction tracking, and project resources tracking in the project management information systems. The blockchains can also be applied for managing assets during projects and ensuring transparency of activities to all stakeholders, so the cost overruns and schedule delays can be reduced. Adopting a blockchain-based smart contract framework can be used for approving the project status reports. However, this research needs to be extended to develop an open-source prototype for the blockchain-based smart contracts, which can measure and evaluate the quality of pre-defined project deliverables. 


\section{Issues in Information Systems}

Volume $X$, Issue $X$, pp. $x$ - $x$, Year

\section{REFERENCES}

Aiping, Z., \& Dongliang, Z. (2016). The Synergy mechanism in University Research Administration Based on Business Process Reengineering Science Research Management, 37(8), 140-145.

Almiani, M., AbuGhazleh, A., Al-Rahayfeh, A., Atiewi, S., \& Razaque, A. (2019). Deep recurrent neural network for IoT intrusion detection system. Simulation Modelling Practice and Theory, 101 (1), 102031

Al-Hasnawi, A., Carr, S. M., \& Gupta, A. (2019). Fog-based local and remote policy enforcement for preserving data privacy in the Internet of Things. Internet of Things, 7(1), 100069.

Ali, S. T., \& Murray, J. (2016). An overview of end-to-end verifiable voting systems. Real-world electronic voting: Design, analysis, and deployment, 173(1), 171-218.

Andoni, M., Robu, V., Flynn, D., Abram, S., Geach, D., Jenkins, D., ... \& Peacock, A. (2019). Blockchain technology in the energy sector: A systematic review of challenges and opportunities. Renewable and Sustainable Energy Reviews, 100(1), 143-174.

Atzori, L., Iera, A., \& Morabito, G. (2010). The internet of things: A survey. Computer networks, 54(15), 27872805.

Bai, Y., Li, Z., Wu, K., Yang, J., Liang, S., Ouyang, B., Chen, Z. \& Wang, J. (2018). “Researchain”: union blockchain-based scientific research project management system. 2018 Chinese Automation Congress (CAC), 1(1), 4206-4209.

Banerjee, M., Lee, J., \& Choo, K. K. R. (2018). A blockchain future for internet of things security: a position paper. Digital Communications and Networks, 4(3), 149-160.

Bayer, D., Haber, S., \& Stornetta, W. S. (1993). Improving the efficiency and reliability of digital time-stamping. In Sequences Ii, Springer, New York, 1(1), 329-334.

Boban, M. (2016). Digital single market and EU data protection reform with regard to the processing of personal data as the challenge of the modern world. Economic and social development: book of proceedings, 16(1), 191.

Brody, P., \& Pureswaran, V. (2014). Device democracy: Saving the future of the internet of things. IBM, September, 1(1), 15.

Buterin, V. (2014). Ethereum: A next-generation smart contract and decentralized application platform. WhitePaper.www.github. com/ethereum/wiki/wiki/\% 5BEnglish\% 5D.

Casado-Vara, R., Chamoso, P., De la Prieta, F., Prieto, J., \& Corchado, J. M. (2019). Non-linear adaptive closedloop control system for improved efficiency in IoT-blockchain management. Information Fusion, 49(1), 227-239.

Casola, V., De Benedictis, A., Riccio, A., Rivera, D., Mallouli, W., \& de Oca, E. M. (2019). A security monitoring system for internet of things. Internet of Things, 7(1), 100080.

Chang, C. H., \& Lai, P. L. (2017). An evaluation of logistics policy enablers between Taiwan and the UK. Maritime Business Review, 2(1), 2-20.

Chen, K. C., \& Lien, S. Y. (2014). Machine-to-machine communications: Technologies and challenges. Ad Hoc Networks, 18(1), 3-23. 


\section{Issues in Information Systems}

Volume $X$, Issue $X$, pp. $x$ - $x$, Year

Christidis, K., \& Devetsikiotis, M. (2016). Blockchains and smart contracts for the internet of things. IEEE Access, 4(1), 2292-2303.

Cong, L. W., \& He, Z. (2019). Blockchain disruption and smart contracts. The Review of Financial Studies, 32(5), 1754-1797.

Delgado-Mohatar, O., Fierrez, J., Tolosana, R., \& Vera-Rodriguez, R. (2020). Blockchain meets Biometrics: Concepts, Application to Template Protection, and Trends [White paper]. BiDAlab. www. github.com/BiDAlab/BlockchainBiometrics.

Deshmukh-Bhosale, S., \& Sonavane, S. S. (2019). A Real-Time Intrusion Detection System for Wormhole Attack in the RPL based Internet of Things. Procedia Manufacturing, 32(1), 840-847.

Di Silvestre, M. L., Gallo, P., Guerrero, J. M., Musca, R., Sanseverino, E. R., Sciumè, G., Vasquez J.C. \& Zizzo, G. (2020). Blockchain for power systems: Current trends and future applications. Renewable and Sustainable Energy Reviews, 119(1), 109585.

Dorri, A., Roulin, C., Jurdak, R., \& Kanhere, S. S. (2019). On the activity privacy of blockchain for IoT. 2019 IEEE 44th Conference on Local Computer Networks (LCN),44(1), 258-261.

Feng, H., Wang, X., Duan, Y., Zhang, J., \& Zhang, X. (2020). Applying blockchain technology to improve agrifood traceability: A review of development methods, benefits, and challenges. Journal of Cleaner Production, 260(1) 121031.

Fernández-Caramés, T. M., \& Fraga-Lamas, P. (2018). A Review on the Use of Blockchain for the Internet of Things. IEEE Access, 6(1), 32979-33001.

Galvez, J. F., Mejuto, J. C., \& Simal-Gandara, J. (2018). Future challenges on the use of blockchain for food traceability analysis. Trends in Analytical Chemistry, 107(1), 222-232.

Giffen, P., (2019). How blockchain will change project management. Available: www.monday.com/blog/projectmanagement/blockchain-will-change-project-management/

Goyal S., (2018). Blockchain technology history: the ultimate guide. [Image] Available: www.101blockchains.com/history-of-blockchain-timeline/\#prettyPhoto.

Groenfeldt, T. (2017). IBM And Maersk Apply Blockchain To Container Shipping. Available: www.forbes.com/sites/tomgroenfeldt/2017/03/05/ibm-and-maersk-apply-blockchain-tocontainer-shipping/\#4a2288eb3f05.

Gronbaek, M. V. H. (2016). Blockchain 2.0, smart contracts, and challenges. Computers \& Law, The SCL Magazine 1(1), $1-5$.

Haber S., Stornetta W.S. (1991) How to Time-Stamp a Digital Document. Menezes A.J., Vanstone S.A. (eds) Advances in Cryptology-CRYPTO' 90. CRYPTO 1990. Lecture Notes in Computer Science, Springer, Berlin, Heidelberg. vol 537(1) 437-445.

Hewavitharana, T., Nanayakkara, S., \& Perera, S. (2019). Blockchain as a project management platform. Proceedings of the 8th World Construction Symposium, 1(1), 137-146.

Huang, X., Xu, C., Wang, P., \& Liu, H. (2018). LNSC: A security model for electric vehicles and charging pile management based on blockchain ecosystem. IEEE Access, 6(1), 13565-13574. 
IP Specialist (2019). How blockchain technology works. Available: www.medium.com/@ipspecialist/howblockchain-technology-works-e6109c033034.

Jogunola, O., Ikpehai, A., Anoh, K., Adebisi, B., Hammoudeh, M., Son, S. Y., \& Harris, G. (2017). State-of-the-art and prospects for peer-to-peer transaction-based energy system. Energies, 10(12), 2106.

Karafiloski, E., \& Mishev, A. (2017). Blockchain solutions for big data challenges: A literature review. IEEE EUROCON 2017-17th International Conference on Smart Technologies, 1(1), 763-768.

Khan, M. A., \& Salah, K. (2018). IoT security: Review, blockchain solutions, and open challenges. Future Generation Computer Systems, 82(1), 395-411.

Kshetri, N. (2018). 1 Blockchain's roles in meeting key supply chain management objectives. International Journal of Information Management, 39 (1), 80-89.

Lacity, M. C. (2018). Addressing key challenges to making enterprise blockchain applications a reality. MIS Quarterly Executive, 17(3), 201-222.

Lawrence, A., (2019). How Blockchain Technology Can Change Project Management. Available: www.orangescrum.com/blog/how-blockchain-technology-can-change-project-management.html?amp

Li, C., Qin, Z., Novak, E., \& Li, Q. (2017). Securing SDN infrastructure of IoT-fog networks from MitM attacks. IEEE Internet of Things Journal, 4(5), 1156-1164.

Li, Y. (2019). Emerging blockchain-based applications and techniques. Service Oriented Computing and Applications, 13(4), 279-285.

Lieber, A. (2017). Trust in trade: Announcing a new blockchain partner. IBM Blockchain. [Online] IBM. Available: www.ibm.com/blogs/blockchain/2017/03/trust-trade-announcing-new-blockchain-partner.

Lohry M. \& Bodell B. (2015). Blockchain-Enabled Co-Housing, Available: www.medium.com/@MatthewLohry/blockchain-enabled-co-housing-de48e4f2b441.

Lund, D., MacGillivray, C., Turner, V., \& Morales, M. (2014). Worldwide and regional internet of things (IoT) 2014-2020 forecast: A virtuous circle of proven value and demand. International Data Corporation (IDC), Tech. Rep, 1(1), 9.

Maesa, D. D. F., \& Mori, P. (2020). Blockchain 3.0 applications survey. Journal of Parallel and Distributed Computing, 138(1), 99-114.

Mazzei, D., Baldi, G., Fantoni, G., Montelisciani, G., Pitasi, A., Ricci, L., \& Rizzello, L. (2020). A Blockchain Tokenizer for Industrial IOT trustless applications. Future Generation Computer Systems, 105(1), 432-445.

Meiklejohn, S., Pomarole, M., Jordan, G., Levchenko, K., McCoy, D., Voelker, G. M., \& Savage, S. (2013). A fistful of bitcoins: characterizing payments among men with no names. In Proceedings of the 2013 conference on Internet measurement conference 127-140.

Microsofr (2019). Summary of research learnings. IoT Signals Available: www.azure.microsoft.com/mediahandler/files/resourcefiles/iot-signals/IoT-Signals-Microsoft-072019.pdf

Mohanta, B. K., Jena, D., Satapathy, U., \& Patnaik, S. (2020). Survey on IoT Security: Challenges and Solution using Machine Learning, Artificial Intelligence, and Blockchain Technology. Internet of Things, 11(1), 100227. 


\section{Issues in Information Systems}

Volume $X$, Issue $X$, pp. $x$ - $x$, Year

Moon, J., Jung, I. Y., \& Park, J. H. (2018). IoT application protection against power analysis attack. Computers \& Electrical Engineering, 67(1), 566-578.

Nakamoto, S. (2008). Bitcoin: A peer-to-peer electronic cash system. Available: www.bitcoin. org/bitcoin. pdf.

Narayanan, A., Bonneau, J., Felten, E., Miller, A., \& Goldfeder, S. (2016). Bitcoin and cryptocurrency technologies: a comprehensive introduction. Princeton University Press.

Nguyen, B. M., Dao, T. C., \& Do, B. L. (2020). Towards a blockchain-based certificate authentication system in Vietnam. PeerJ Computer Science, 6(1), e266.

Nguyen, H. P. (2019). Blockchain-an indispensable development trend of the logistics industry in Vietnam: Current situation and recommended solutions. Int. JJ e-Navigation e-Navigation Marit. Econ, 13(1), 14-22.

Norris, T., (2019). Using Blockchain as a Project Management tool. Available: www.ibm.com/blogs/blockchain/2019/06/using-blockchain-as-a-project-management-device/

Olsen, P., \& Borit, M. (2018). The components of a food traceability system. Trends in food science \& technology, 77(1), 143-149.

Pan, Y., Zhang, X., Wang, Y., Yan, J., Zhou, S., Li, G., \& Bao, J. (2019). Application of blockchain in carbon trading. Energy Procedia, 158(1), 4286-4291,

Pawczuk, L., Massev, R., \& Schatsky, D. (2018). Breaking blockchain open Deloitte’s 2018 global blockchain survey. Deloitte. Available: www.deloitte.com/content/dam/Deloitte/us/Documents/financial-services/usfsi-2018-global-blockchain-survey-report.pdf

Perrons, R. K., \& Cosby, T. (2020). Applying blockchain in the geo-energy domain: The road to interoperability and standards. Applied Energy, 262(1), 114545.

Pop, C., Cioara, T., Anghel, I., Antal, M., \& Salomie, I. (2020). Blockchain-based Decentralized Applications: Technology Review and Development Guidelines.

Pournader, M., Shi, Y., Seuring, S., \& Koh, S. L. (2020). Blockchain applications in supply chains, transport, and logistics: a systematic review of the literature. International Journal of Production Research, 58(7), 20632081.

Prada-Delgado, M. Á., Baturone, I., Dittmann, G., Jelitto, J., \& Kind, A. (2020). PUF-derived IoT identities in a zero-knowledge protocol for blockchain. Internet of Things, 9(1), 100057.

Raj R., (2020) How does blockchain work Available: www.intellipaat.com/blog/tutorial/blockchain-tutorial/howdoes-blockchain-work/

Rassenfoss, S. (2018). Can Putting Blockchain on Drilling Rigs Really Get Everyone on the Same Screen?. Journal of Petroleum Technology, 70(09), 44-46.

Reyna, A., Martín, C., Chen, J., Soler, E., \& Díaz, M. (2018). On blockchain and its integration with IoT. Challenges and opportunities. Future generation computer systems, 88(1), 173-190.

Roman, R., Zhou, J., \& Lopez, J. (2013). On the features and challenges of security and privacy in distributed internet of things. Computer Networks, 57(10), 2266-2279.

Sadique, K. M., Rahmani, R., \& Johannesson, P. (2018). Towards security on the internet of things: applications and challenges in technology. Procedia Computer Science, 141(1), 199-206. 


\section{Issues in Information Systems}

Volume $X$, Issue $X$, pp. $x$ - $x$, Year

Sengupta, J., Ruj, S., \& Bit, S. D. (2020). A Comprehensive survey on attacks, security issues, and blockchain solutions for IoT and IIoT. Journal of Network and Computer Applications, 149(1), 102481.

Szabo, N. (1997). The idea of smart contracts. Nick Szabo’s Papers and Concise Tutorials, 6(1).

Siddiqui, I., (2018) What the Hell Is Blockchain and How Does It Work (Simplified) Available: www.medium.com/coinmonks/what-the-hell-is-blockchain-and-how-does-it-works-simplifiedb9372ecc26ef.

Thakur, M., \& Donnelly, K. A. M. (2010). Modeling traceability information in soybean value chains. Journal of Food Engineering, 99(1), 98-105.

Turk, Ž. \& Klinc, R. (2017). Potentials of blockchain technology for construction management. Procedia engineering, 196(1), 638-645.

Wang, Q., Zhu, X., Ni, Y., Gu, L., \& Zhu, H. (2019). Blockchain for the IoT and Industrial IoT: A review. Internet of Things, 1(1), 100081.

Wolf, P., Nackerdien, R., \& Tuccinardi, D. (2011). Introducing electronic voting: essential considerations. International Institute for Democracy and Electoral Assistance (International IDEA).

Wurm, J., Hoang, K., Arias, O., Sadeghi, A. R., \& Jin, Y. (2016). Security analysis on consumer and industrial IoT devices. 21st Asia and South Pacific Design Automation Conference (ASP-DAC), 1(1), (519-524).

Yang, C. S. (2019). Maritime shipping digitalization: Blockchain-based technology applications, future improvements, and intention to use. Transportation Research Part E: Logistics and Transportation Review, 131(1), 108-117.

Xiao S. \& Hao Y. (2016). Innovative on Risk Prevention and Control and Optimization Mechanism of Research Projects. Process Management Science and Technology Management Research. 36(13), 176-180.

Zhao, G., Liu, S., \& Lopez, C. (2017). A literature review on risk sources and resilience factors in agri-food supply chains. Working Conference on Virtual Enterprises, 506(1), 739-752.

Zhao, H., Zhang, Y., Peng, Y., \& Xu, R. (2017). Lightweight backup and efficient recovery scheme for health blockchain keys. 13th International Symposium on autonomous decentralized system (ISADS), 1(1), 229234.

Zheng C., \& Bai Y., (2018). Brief Discussion on Scientific Research Project Management using Blockchain. Journal of Guiyang University- Natural Sciences. 13(2), 48-51. 